Chirurg 2016 $87: 1039-1045$

DOI 10.1007/s00104-016-0300-4

Online publiziert: 12. Oktober 2016

(c) Der/die Autor(en) 2016. Dieser Artikel ist

eine Open-Access-Publikation.

CrossMark

\author{
R. Pabst ${ }^{1}$ P. B. Linke ${ }^{2}$ N. B. A. Neudeck ${ }^{2}$ M. Schmiel $\left.\right|^{3}$ S. B. Ernst ${ }^{4}$ \\ ' Institut für Immunmorphologie Zentrum Anatomie, Medizinische Hochschule Hannover, Hannover, \\ Deutschland \\ ${ }^{2}$ Forschungsdekanat, Medizinische Hochschule Hannover, Hannover, Deutschland \\ ${ }^{3}$ Bibliothek, Medizinische Hochschule Hannover, Hannover, Deutschland \\ ${ }^{4}$ Institut für Biometrie, Medizinische Hochschule Hannover, Hannover, Deutschland
}

\title{
Anschubfinanzierung für junge Wissenschaftler in der Universitätsmedizin in Deutschland
}

\section{Ist der "clinical scientist“ so zu retten?}

\section{Zusatzmaterial online \\ Die Onlineversion dieses Beitrags (doi: 10.1007/s00104-016-0300-4) enthält den Fragebogen zur hochschulinternen Leistungsförderung (HILF) der Medizi- nischen Hochschule Hannover. Beitrag und Zusatzmaterial stehen Ihnen im elektronischen Volltextarchiv auf http:// www.springermedizin.de/der-chirurg zur Verfügung. Sie finden das Zusatzmaterial am Beitragsende unter "Supplementary Material“.}

\section{Fragestellung}

Die medizinischen Fakultäten in Deutschland leiden unter wachsenden Finanzierungsproblemen, wie sich an den z.T. hohen Defiziten im Jahr 2013 ablesen lässt. Wesentliche Gründe dafür liegen im Sonderstatus der Unikliniken als Krankenhäuser der Maximalversorgung, ihrer Schlüsselrolle in der ärztlichen Weiterbildung, der Behandlung seltener und oft sehr teurer Erkrankungen sowie der parallel durchgeführten Lehrveranstaltungen für die Studierenden, wie dies Albrecht et al. [1] und Bitzinger et al. [6] 2013 und 2014 in der Zeitschrift Deutsches Ärzteblatt zusammenfassten.

R. Pabst und P.B. Linke haben zu gleichen Teilen zu der Arbeit beigetragen.
Die Promotion ist für zukünftige Wissenschaftler weiterhin wichtig und sollte möglichst schon im Studium erfolgen, um dafür keine Zeit in der Weiterbildungsphase aufwenden zu müssen $[3,5,22]$. Diese Forderung gewinnt Bedeutung vor dem Hintergrund, dass nur promovierte Wissenschaftler einen Antrag im Normalverfahren der Deutschen Forschungsgemeinschaft (DFG) stellen dürfen. Diesen Umstand haben Kroemer und Kawczek [19] kürzlich zum Anlass genommen, um auf die Notwendigkeit einheitlicher Infrastrukturen hinzuweisen. So kann derzeit die Drittmittelforschung nicht nur als Garant, sondern auch als Gefahr für die Forschungsfreiheit angesehen werden [12]. Besondere Bedeutung kommt aber auch dem Umstand zu, engagierte Personen rechtzeitig für die Forschungsarbeit zu motivieren. An der Forschung interessierte Medizinstudenten sind durch spezielle Angebote, wie das Biomedical Exchange Program (BMEP) erreichbar [4]. Das Problem der Motivation zur aktiven Forschungsbeteiligung liegt nicht zuletzt an der langen Ausbildungszeit für Ärzte, die nach einem sechsjährigen Medizinstudium meist noch eine fünfährige Weiterbildung (oft sind für eine Zusatzbezeichnung, zB. Gastroenterologie, weitere Fortbildungsjahre notwendig) absol- vieren müssen. In der Chirurgie beträgt die Weiterbildungszeit sogar 6 Jahre.

Auf einen drohenden Mangel an Chirurgen hat Ansorg 2012 [2] mit Nachdruck hingewiesen. In einer Evaluation des Blockpraktikums Chirurgie und des Abschnittes des Praktischen Jahres in der Chirurgie fühlten sich Studentinnen und Studenten vergleichbar gut betreut und trotzdem planten weniger Frauen eine chirurgische Weiterbildung, ohne dass dafür Gründe angegeben wurden [24].

Männer und Frauen unterscheiden sich wesentlich im Hinblick auf Karrierewünsche, weil Frauen ihre Karrierewünsche hinter die Familie stellen, dies belegt eine Schweizer Studie an Staatsexamenskandidaten [7].

Im Herbst 2015 wurde ein dringender Aufruf in den USA publiziert, der die Existenz des „clinical scientist“"als extrem gefährdet sieht [21]. Die Prognosen für Deutschland sehen nicht besser aus, wie kürzlich Epstein et al. ausführlich dokumentiert haben [14]. Deutliche Unterschiede zwischen Männern und Frauen u. a. bei der Publikation der Egebnisse der Medizinischen Promotion belegte kürzlich Pfeffer et al [25]. Studierende der Medizin sollten bereits im Studium mehr Kontakt mit der Forschung haben, wie es durch eine strukturierte Doktoran- 


\section{Aktuelles Thema}

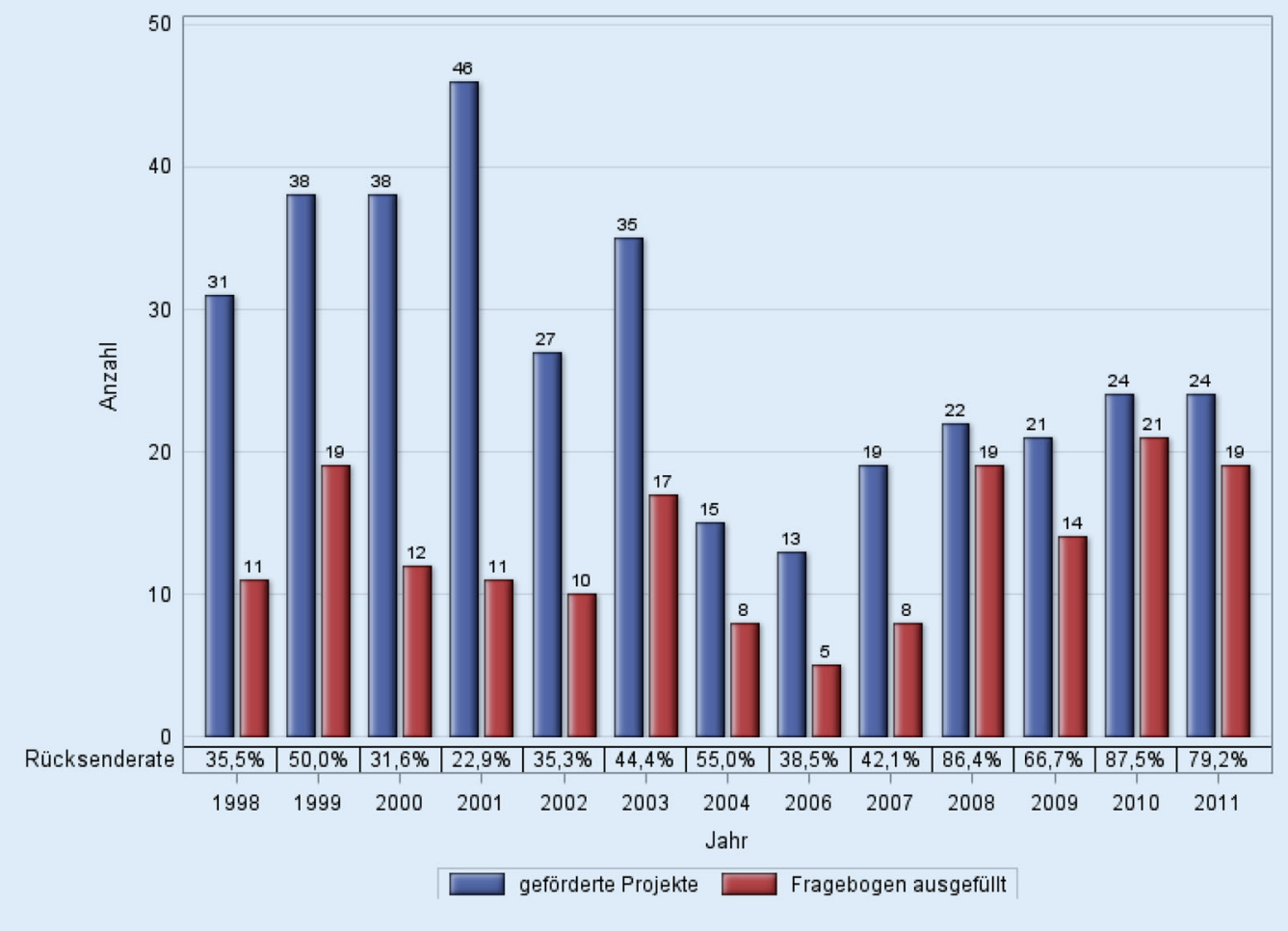

Abb. $1<$ Beteiligung an der Befragung

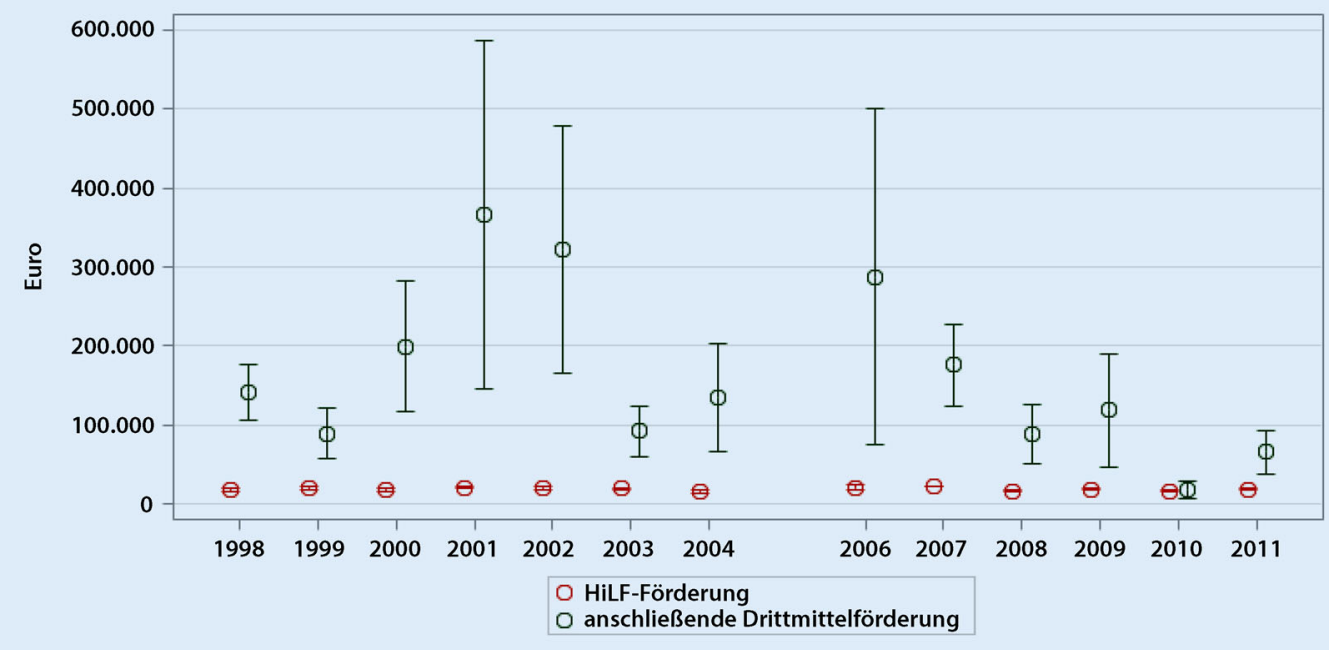

Abb. $2 \triangleleft$ Durchschnittliche Ausgaben und Einnahmen pro Projekt. HiLF Fragebogen zur hochschulinternen Leistungsförderung

denausbildung möglich ist, wie kürzlich von Pfeiffer et al. belegt [23].

In einer Umfrage unter den Dekanen der medizinischen Fakultäten in Deutschland $\mathrm{zu}$ ihren internen Programmen der Nachwuchsförderung in der Forschung bestätigten $91 \%$ der angeschriebenen Fakultäten, dass zahlreiche oft unterschiedliche Programme im Hinblick auf deren Zielgruppe, Förderdauer und Förderhöhe z. B. Promotionsstipendien, Startfinanzierung einer eigenen
Forschergruppe [23] existieren, jedoch keine Fakultät ihre Programme bisher evaluiert hat. Aus diesem Grund sollen hier die Ergebnisse der Evaluation der hochschulinternen leistungsbezogenen Forschungsförderung (HILF) an der Medizinischen Hochschule Hannover $(\mathrm{MHH})$ dargestellt werden. Dabei stehen folgende Kriterien im Vordergrund: 1. Wie erfolgte die Auswahl der geförderten Projekte?
2. Werden die wissenschaftlichen Ziele wie Publikationen und anschließende Drittmitteleinwerbungen erreicht?

3. Gibt es Unterschiede zwischen Männern und Frauen?

\section{Methodik}

Im Jahr 1998 wurde aufgrund eines Vorschlags der Forschungskommission das HILF-Programm an der MHH eingeführt. Zweimal jährlich können pro- 
Chirurg 2016 :87:1039-1045 DOI 10.1007/s00104-016-0300-4

(c) Der/die Autor(en) 2016. Dieser Artikel ist eine Open-Access-Publikation.

R. Pabst • P. B. Linke - N. B. A. Neudeck · M. Schmiel · S. B. Ernst

\section{Anschubfinanzierung für junge Wissenschaftler in der Universitätsmedizin in Deutschland. Ist der „clinical scientist" so zu retten?}

\section{Zusammenfassung}

Hintergrund. An den medizinischen Fakultäten in Deutschland konkurrieren Krankenversorgung, Lehre und Forschung um finanzielle Mittel. Dabei droht die Gefahr, dass aufgrund der prekären Finanzlage der Fakultäten eine Anschubfinanzierung der Forschung junger Postdoktoranden gekürzt oder überhaupt nicht mehr möglich ist. Dieser Umstand war Anlass, um die Ergebnisse der hochschulinternen Anschubfinanzierung an der Medizinischen Hochschule Hannover (MHH) zu evaluieren, was bisher an keiner deutschen Hochschule geschehen ist. Methode. Die Empfänger der hochschulinternen leistungsbezogenen Förderung (HILF) der Förderjahrgänge 1998 bis 2011 wurden zum Umfang der Förderung, zur anschließenden Drittmittelförderung durch die Deutsche Forschungsgemeinschaft (DFG) oder Stiftungen sowie zur Anzahl der erfolgten Publikationen befragt. Die Antworten wurden insgesamt sowie nach dem Geschlecht der Antragsteller ausgewertet.

Ergebnisse. Grundsätzlich ist festzustellen, dass sich die chirurgischen Fächer nicht von denen der inneren Medizin unterscheiden. Die Anzahl aller eingereichten Anträge schwankte zwischen 28 und 96 pro Förderjahrgang, wobei die Bewilligungsquote in den Jahren 1998 bis 2004 bei $69,5 \pm 14,0 \%$ und in den Jahren 2006 bis 2011 bei 30,9 $\pm 11,0 \%$ pro Jahr lag. In diesem Zeitraum wurden insgesamt 353 Projekte gefördert. Für die Evaluation lagen 174 (49,2\%) beantwortete und auswertbare Fragebögen vor. Das mittlere Fördervolumen betrug 18.640 EUR pro Projekt.
Die im Anschluss eingeworbenen Drittmitte von der DFG oder Stiftungen betrugen im Mittel das 7,2-Fache der von der Hochschule eingesetzten Mittel. Inzwischen haben sich $56 \%$ der Männer und $42 \%$ der Frauen habilitiert. Insgesamt $71 \%$ der Befragten bezeichnen die Anschubfinanzierung als hilfreich für die Habilitation.

Schlussfolgerungen. Das Programm der hochschulinternen Anschubfinanzierung der Forschung für Postdoktoranden ist insgesamt erfolgreich.

\section{Schlüsselwörter}

Medizinische Forschung · Anschubfinanzierung · Postdoktoranden · Evaluation

\section{Start-up grants for young scientists in German medical universities. Can the clinical scientist be saved?}

\section{Abstract}

Background. German medical faculties currently have severe financial problems. There is the conflict between financing teaching medical students, inpatient and outpatient costs and supporting basic and applied research.

Methods. Young postdocs can apply for a grant to start research projects to establish techniques on publishing data as a basis for applying for grants from the German Research Foundation or foundations with a critical review system. Successful applicants from the years 1998-2011 were asked to answer a questionnaire.
Results. The annual number of applications ranged from 28 to 96 per year. Within the first period of our analysis ranging from 1998 to 2004 , a mean number of $69.5 \% \pm$ $14.0 \%$ of submitted grant applications were approved annually in comparison to an average approval of $30.9 \% \pm 11 \%$ in the years 2006-2001. In total 353 projects were funded with a mean amount of money for a project of approximately 18,640 EUR. The mean amount of external grant money following the start-up period was 7.2 times the money initially spent. That is an excellent return of investment. There were no differences between applicants from the department of surgery or department of internal medicine. In the meantime, $56 \%$ of men and $42 \%$ of women have achieved the academic degree university lecturer (privatdozent). Furthermore, $71 \%$ of the participants evaluated this start-up research as supportive for their postdoctoral qualification (habilitation).

Conclusion. The program for initial investment for young postdocs by internal start-up grants is overall successful.

\section{Keywords}

Start-up grants - Medical research . Postdoctoral scientists · Evaluation movierte Wissenschaftler seitdem einen Antrag auf Förderung eines eigenen Forschungsprojektes mit einer Gesamtfördersumme von maximal 25.000 EUR stellen. Eine Altersbegrenzung besteht dabei nicht. Die Mittel können für Personal- und Sachkosten, nicht aber für Reisen oder elektronische Hilfsmittel (z. B. Computer) eingesetzt werden. Die Laufzeit beträgt maximal ein Jahr. Von der Forschungskommission bewertete und bewilligte Anträge sind an die Verpflichtung gebunden, einen Ab- schlussbericht in Verbindung mit einem Vortrag bzw. einem Poster zu erstellen. Der beste Vortrag und das beste Poster werden jährlich mit je 500 EUR von der Gesellschaft der Freunde der $\mathrm{MHH}$ prämiert. Im Jahr 2005 wurde aus finanziellen Gründen kein HILF-Programm ausgeschrieben. Im Übrigen gab es für einige Jahre ein Zusatzprogramm zur Förderung von Kooperationsprojekten (genannt HILF II), das aber aus finanziellen Gründen ohne Evaluation wieder eingestellt wurde. Die vorliegenden Da- ten beziehen sich daher nur auf das ursprüngliche HILF-Programm. Für die Evaluation wurden alle Projektleiter der Jahrgänge 1998 bis 2011 angeschrieben und deren zurückgesandten Antworten ausgewertet. Der Fragebogen umfasste die Teilbereiche Publikation, Vorträge und Poster sowie bewilligte Drittmittelprojekte auf der Grundlage des HILFProjekts. Die Angabe der Publikationen wurde durch Ermittlung der ImpactFaktoren objektiviert. Der Fragebogen 


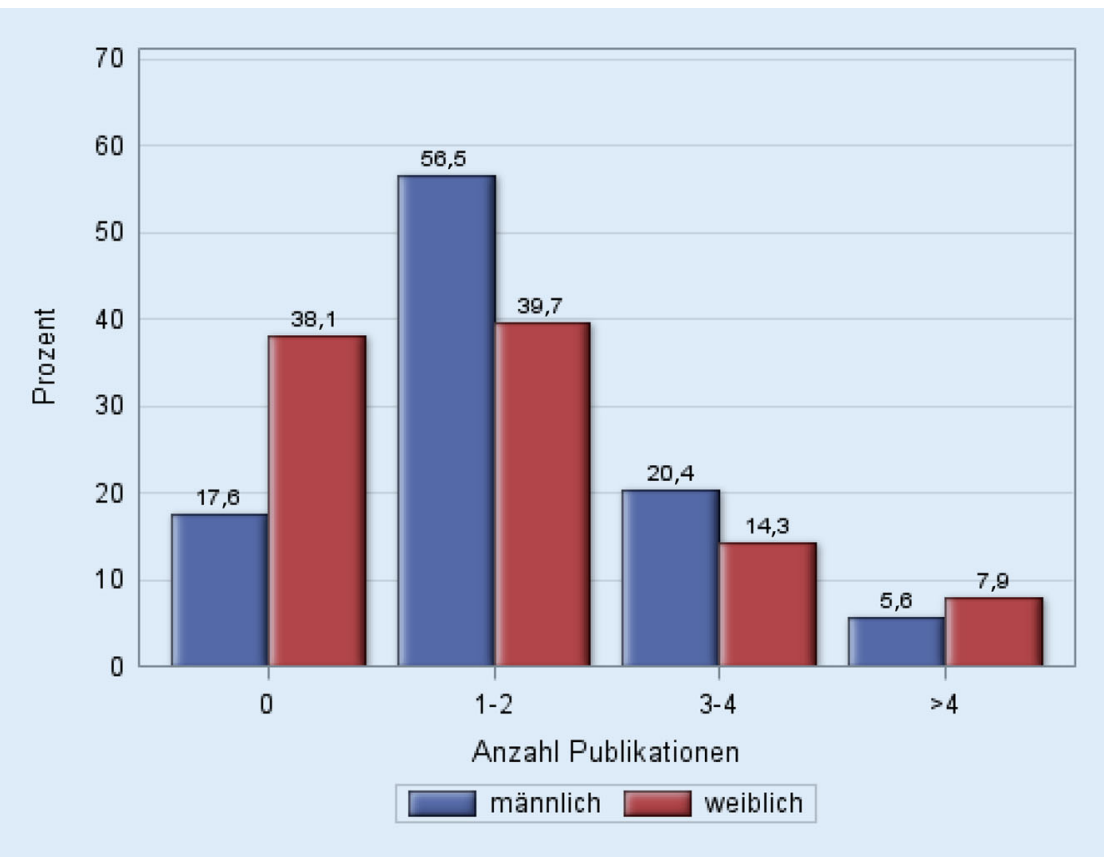

Abb. $3 \Delta$ Anzahl Publikationen nach Geschlecht

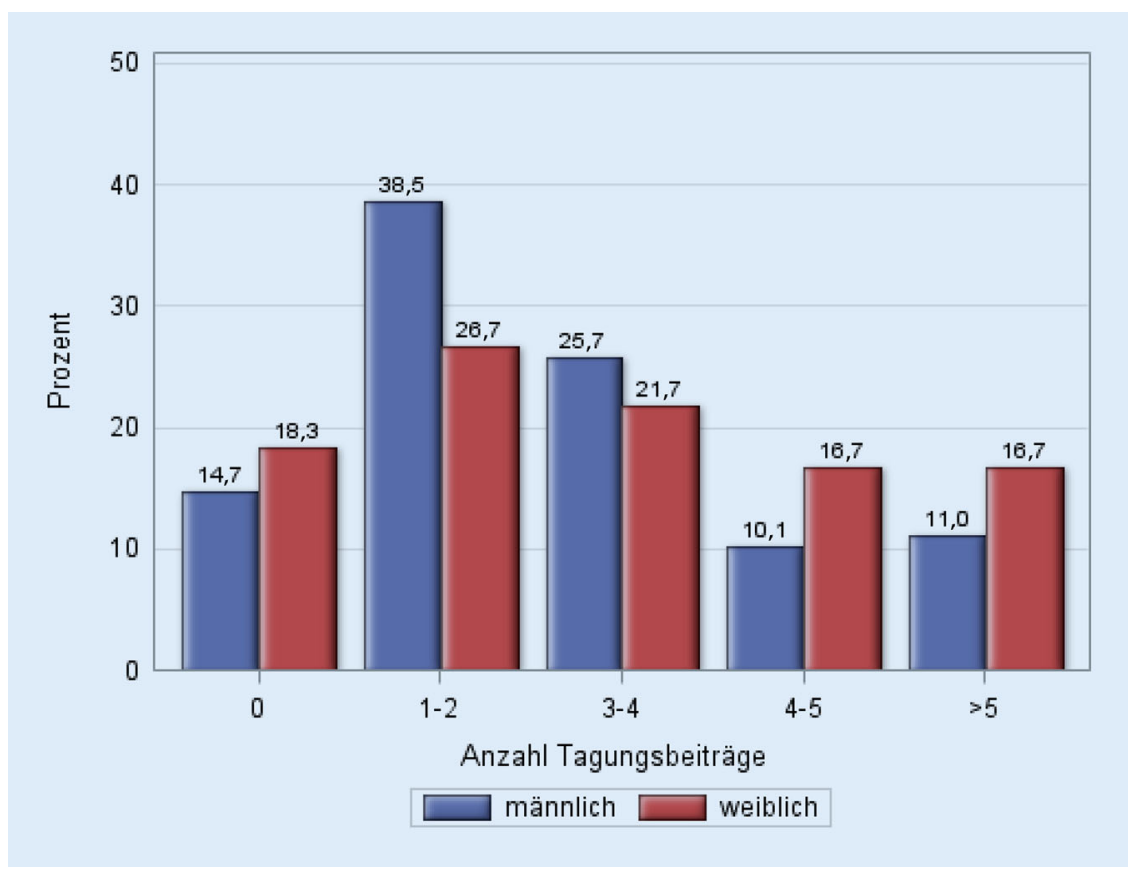

Abb. 4 \& Anzahl der Tagungsbeiträge nach Geschlecht

steht als elektronischer Anhang dieser Arbeit zur Verfügung.

\section{Resultate}

Von 1998 bis 2011 wurden insgesamt 353 Projekte nach kritischer Begutachtung durch die Forschungskommission gefördert, wobei die Anzahl der eingereich-
Ausgefüllte und auswertbare Fragebogen wurden von 174 (49,2\%) Geförderten zurückgesandt. Es bestanden keine Unterschiede in der Autorenquote zwischen Geförderten aus der inneren Medizin (34\%) und Chirurgie (35\%). Die Rücklaufquote variierte in den einzelnen Förderjahrgängen (• Abb. 1), wobei die von 1998 bis 2007 geförderten Projektleiter sich deutlich weniger an der Befragung beteiligten als die der nachfolgenden Jahre. Das durchschnittliche Alter lag bei 33,7 \pm 4,2 Jahre. Die Höhe der eingeworbenen Drittmittel betrug durchschnittlich das 7,2-Fache der durch die Hochschule eingesetzten Fördermittel. Dabei zeigten sich deutliche Unterschiede je nach Förderjahrgang (- Abb. 2). Die Nationalität der Rücksender war zu $92 \%$ deutsch. Von den 50 geförderten Projektträgern, die sich vor ihrem bewilligten HILF-Antrag im Ausland aufhielten, waren über zwei Drittel in den Vereinigten Staaten von Amerika. Auffällig ist, dass der Anteil weiblicher Projektträger ohne Publikation $38 \%$ aus dem HILF-geförderten Projekt im Vergleich zu männlichen Projektträgern $17,6 \%$ beträgt (• Abb. 3 ). Es wurden auch zahlreiche Tagungsbeiträge mit Daten aus den HILF-Projekten dokumentiert (• Abb.4). Sowohl $80 \%$ der Männer als auch der Frauen haben hier mindestens einen Tagungsbeitrag eingereicht. Die Anschlussförderung variierte zwischen den Förderjahrgängen, liegt aber im Schnitt bei einer guten Förderungsrate von 56,3\% (• Abb. 5). Bei der Anzahl der Patentanmeldungen, die aus ca. $6 \%$ der geförderten Projekte hervorgingen, gab es keinen Geschlechtsunterschied. Die wenigen Frauen hatten im Vergleich zu den männlichen Projektanträgern im Mittel größere Impact-Faktoren der auf dem HILF-Projekt basierenden Publikationen erhalten (Männer: 3,8 \pm 3,7; Frauen: 4,5 $\pm 3,3 ; p=0,0950$, t-test; • Tab. 1). Mindestens eine Anschlussfinanzierung erhielten 62,7 \% der Männer und 43,3 \% der Frauen ( $p=0,0245$, Binomial-Test). Da oft Anträge auf Anschlussförderung erst nach einer erfolgreichen Publikation sinnvoll sind, haben wir uns auf eine Zeit bis 2011 beschränkt. Bei der Anzahl der Folgeförderung durch die DFG und Stiftungen mit kritischem Reviewsys- 


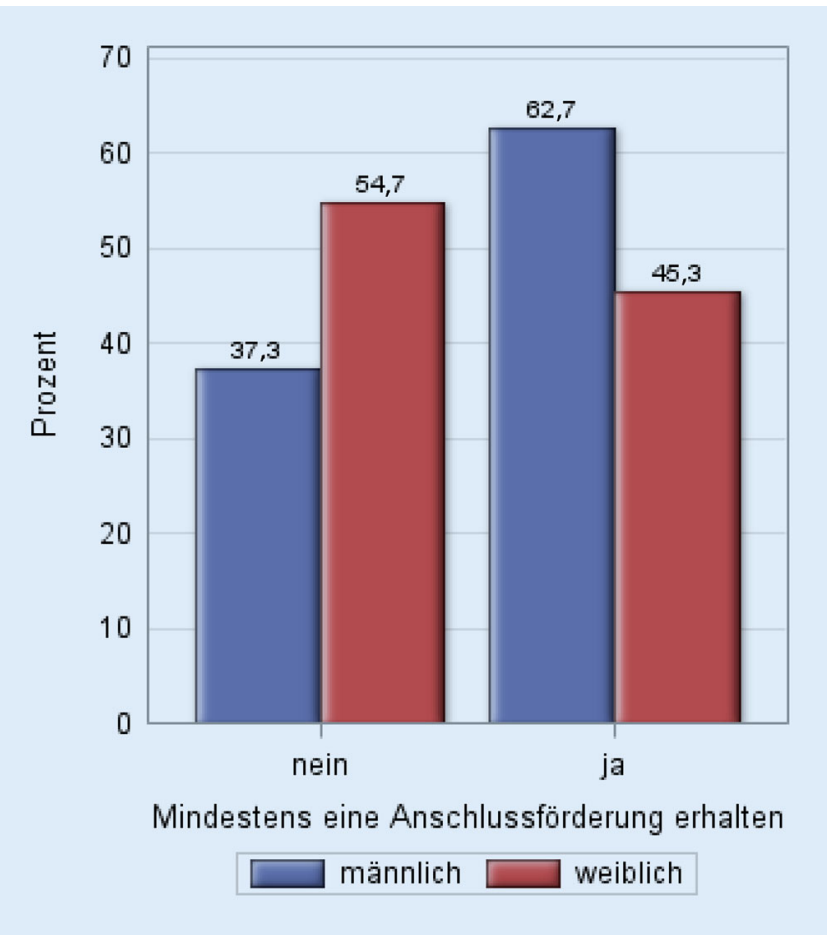

Abb. $5<$ Anschlussförderung

tem bestand kein Unterschied zwischen den Geschlechtern, während bei der Förderung durch sonstige Förderer die Männer mit 28,2 \% erfolgreicher als die Frauen mit $14,1 \%$ waren $(p=0,0208$, Binomial-Test). Inzwischen haben sich $56 \%$ der befragten Männer und $42 \%$ der Frauen habilitiert, wobei $71 \%$ angaben, dass das HILF-Projekt relevant für ihre bereits erfolgreiche bzw. noch andauernde Habilitation gewesen sei. Die freien Äußerungen waren zum überwiegenden Teil positiv. Die Förderung durch HILF im Verhältnis zu allen Drittmitteleinnahmen schwankt zwischen den Kliniken, aber es bestanden keine signifikanten Unterschiede zwischen den sieben Kliniken des Zentrums für Innere Medizin und den sieben Kliniken des Zentrums Chirurgie. Wenn die Ergebnisse in der Chirurgie und Inneren Medizin pro Klinik dargestellt werden (• Abb. 6) zeigen sich Unterschiede zwischen den Kliniken.

Wiederholt wurde um eine Aufstockung und Erweiterung des Programms gebeten. Für die hochschulinterne Diskussion in der Forschungskommission wurden die Ergebnisse nach operativen/ nichtoperativen, vorklinischen/klinisch theoretischen Fächern und der psychologischen Medizin getrennt ausgewertet.

\section{Diskussion}

Die Ergebnisse dieser Befragung zeigen die Effektivität des Programms zur Anschubförderung der Forschung für junge Wissenschaftler. Allerdings sollte einschränkend festgehalten werden, dass keine Vergleichsgruppe vorlag. Einige nicht geförderte Antragsteller waren nicht bereit, an der Befragung teilzunehmen, was dazu führte, dass diese Gruppe nicht weiter verfolgt wurde. Bei der Evaluation haben wir nicht zwischen Medizinern und Naturwissenschaftlern unterschieden. Bei den Bewilligungssummen pro Klinik muss berücksichtigt werden, dass sich die Zahlen der wissenschaftlichen Mitarbeiter unterscheiden und Wechsel der Klinikleitung sicher auch einen Einfluss haben. Die Gründe für die unterschiedlichen Bewilligungsquoten in den Jahren 1998 bis 2004 und 2006 bis 2011 sind nicht bekannt. Die Zusammensetzung der Auswahlkommission hängt natürlich von der Wahlperiode der akademischen Gremien ab. Die Tatsache der Unterschiede wurde erst nach dieser Evaluation auch für uns deutlich. Der geringe Datenrücklauf von Personen, die vor langer Zeit gefördert wurden, überrascht nicht, da viele von ihnen schon nicht mehr an der $\mathrm{MHH}$
Tab. 1 Impact-Faktoren der Publikatio-

nen nach Geschlecht

\begin{tabular}{|c|c|c|}
\hline Geschlecht & $\begin{array}{l}\text { Männlich } \\
\text { ( } n=179)\end{array}$ & $\begin{array}{l}\text { Weiblich } \\
(n=98)\end{array}$ \\
\hline Ohne & $\begin{array}{l}22 / 177 \\
(12,4 \%)\end{array}$ & $\begin{array}{l}10 / 98 \\
(10,2 \%)\end{array}$ \\
\hline$>0-<5$ & $\begin{array}{l}111 / 177 \\
(62,7 \%)\end{array}$ & $\begin{array}{l}51 / 98 \\
(52,0 \%)\end{array}$ \\
\hline$\geq 5-<10$ & $\begin{array}{l}35 / 177 \\
(19,8 \%)\end{array}$ & $\begin{array}{l}26 / 98 \\
(26,5 \%)\end{array}$ \\
\hline$\geq 10-<15$ & $\begin{array}{l}7 / 177 \\
(4,0 \%)\end{array}$ & $\begin{array}{l}11 / 98 \\
(11,2 \%)\end{array}$ \\
\hline$\geq 15$ & $\begin{array}{l}2 / 177 \\
(1,1 \%)\end{array}$ & $\begin{array}{l}0 / 98 \\
(0,0 \%)\end{array}$ \\
\hline \multicolumn{3}{|c|}{ Impactfactor 0,0615 ( $x^{2}$-Test) } \\
\hline
\end{tabular}

tätig sind. Ein wesentlicher Vorteil des Programms ist die Eigenverantwortlichkeit und Flexibilität bei der Ausgabe der bewilligten Mittel. Junge Wissenschaftler sind als Postdoktoranden sonst nur in größere Forschungsprojekte eingebunden und haben dabei weder eine eigene Verantwortung noch Berichtspflicht. Allerdings müssen die Belastungen durch Weiterbildung bei Medizinern, die in der Lehre tätig sind, berücksichtigt werden. Trotzdem muss dem Programm im Hinblick auf eine Habilitation der Geförderten eine zunehmende Bedeutung zugeschrieben werden. In einer kürzlich publizierten Studie zur Habilitation in der Medizin wurde der Wunsch nach finanzieller Förderung von $40 \%$ der Befragten geäußert [27]. Es darf nicht vergessen werden, dass das Privatleben (Stichwort Generation Y) und die berufliche Qualifikation in der Forschung miteinander in Einklang gebracht werden müssen [26]. Natürlich sind Impact-Faktoren nur ein Parameter und für eine umfangreiche Bewertung der Forschungsleistungen sollte man umfangreichere Bewertungen einbeziehen sowie kürzlich Herrmann-Lingen et al. [18] betonten und Epstein et al. aktuell belegen [14]. Die an der MHH angebotenen regelmäßigen Veranstaltungen zur erfolgreichen Antragstellung für die Erlangung von Drittmitteln, ein spezielles Mentoring-Programm für Frauen in der Wissenschaft [20] und das HILFProgramm bilden solide Bausteine zur Unterstützung der medizinischen Forschung. Alle diese Maßnahmen sind 


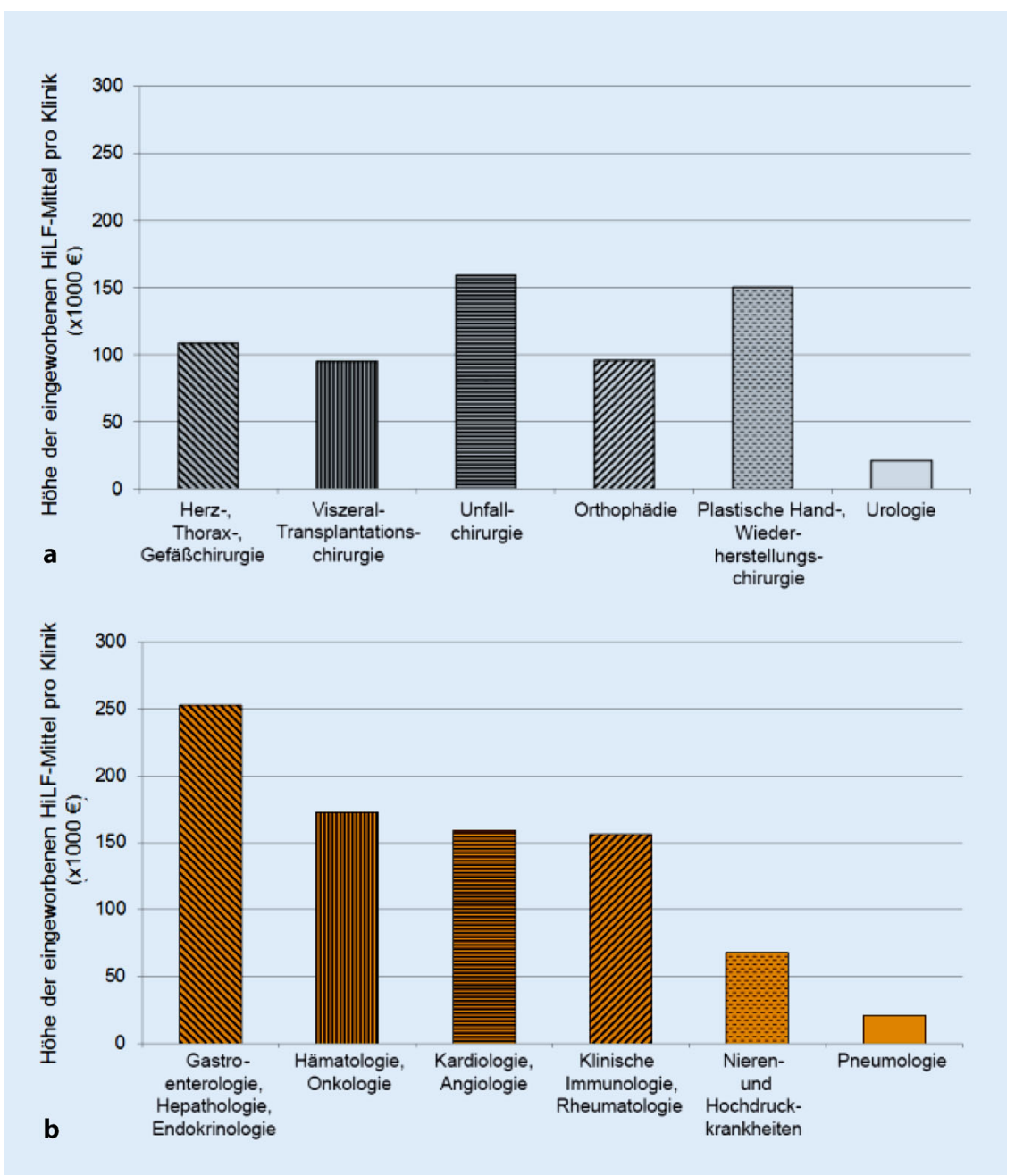

Abb. 6 \ Höhe der eingeworbenen HilF-Gelder pro Klinik in a Department Chirurgie und b Department Innere Medizin (1000 E). HiLF Fragebogen zur hochschulinternen Leistungsförderung

wahrscheinlich ein Grund für die überproportional erfolgreich gestellten Drittmittelanträge bei der DFG im Vergleich $\mathrm{zu}$ anderen medizinischen Fakultäten [9, 11]. Trotz aktueller finanzieller Probleme mit denen alle medizinischen Fakultäten kämpfen [1, 15, 17], darf die jetzige Generation der Wissenschaftler in der jungen Postdoktorandenphase nicht alleine gelassen werden. Damit können nicht nur die Grundlage zur erfolgreichen Einwerbung von Forschungsmitteln gelegt und eine Motivation für junge Wissenschaftler geschaffen werden, sondern auch Belastungen durch Facharztweiterbildung und Aufgaben in der studentischen Lehre überwunden werden [14, 17]. Klinikdirektoren sollten Mitarbeiter nachdrücklich auf die jeweilige Möglichkeit der Forschungsförderung der eigenen Fakultät wie der

\section{Fazit für die Praxis}

Das HILF-Programm ist eine mögliche Lösung dieses Problems. Wir würden es begrüßen, wenn sich der Medizinische Fakultätentag mit Programmen zur Anschubfinanzierung befasst, und möchten alle Fakultäten anregen, ihre Fördermöglichkeiten für junge Wissenschaftler kritisch zu evaluieren. Dann könnten aus den Erfahrungen der anderen Fakultäten für die eigene Fakultät sinnvolle Hinweise erhalten werden, um weiterhin Nachwuchs als "clinical scientist" zu rekrutieren.

\section{Korrespondenzadresse}

\section{Prof. Dr. med. R. Pabst}

Institut für Immunmorphologie Zentrum Anatomie, Medizinische Hochschule Hannover Carl-Neuberg-Str.1, 30625 Hannover,

Deutschland

pabst.reinhard@mh-hannover.de

Danksagung. Der Gesellschaft der Freunde der MHH sei für eine finanzielle Unterstützung des Projekts gedankt. Frau S. Wallbaum danken wir für die Hilfe beim Erstellen des Manuskripts, Frau K. Flunkert bei der Statistik und Frau M. Peter bei den Abbildungen.

\section{Einhaltung ethischer Richtlinien}

Interessenkonflikt. R. Pabst, P. B. Linke, N. B. A. Neudeck, M. Schmiel und S. B. Ernst geben an, dass kein Interessenkonflikt besteht.

Dieser Beitrag beinhaltet keine von den Autoren durchgeführten Studien an Menschen oder Tieren.

oder Gerok Stelle) aufmerksam machen [23] und individuelle Freiräume für interessierte Ärzte und Ärztinnen schaffen. Das gilt besonders für Frauen bei einem steigenden Prozentsatz der jungen Ärztinnen [7, 8]. Die ständige Senatskommission für Grundsatzfragen in der klinischen Forschung der DFG hat im Juni 2014 nachdrücklich auf die aktuellen Probleme hingewiesen [10] und die Expertenkommission für Forschung und Innovation hat den Handlungsbedarf betont [15]. Eine aktuelle Studie der Else-Kröner-Fresenius-Stiftung dokumentiert die derzeit schwierigen Rahmenbedingungen medizinischer Forschung in Deutschland [13].
Open Access. Dieser Artikel wird unter der Creative Commons Namensnennung 4.0 International Lizenz (http://creativecommons.org/licenses/by/4.0/deed. de) veröffentlicht, welche die Nutzung, Vervielfältigung, Bearbeitung, Verbreitung und Wiedergabe in jeglichem Medium und Format erlaubt, sofern Sie den/die ursprünglichen Autor(en) und die Quelle ordnungsgemäßnennen, einen Linkzur Creative Commons Lizenz beifügen und angeben, ob Änderungen vorgenommen wurden.

\section{Literatur}

1. Albrecht M, Kroemer HK, StrehI R (2013) Finanzierung der Universitätsmedizin: An der Grenze der Belastbarkeit. Dtsch Ärztebl 110:A65-A 68

2. Ansorg J, Krüger M, Vallböhmer D (2012) Sinnvolle Weiterbildungskonzepte für Chirurgen. Chirurg 83:360-367

3. Baum C, Förster R, Schmidt RE (2009) Weiterentwicklung des Promotionsverfahrens in der Medizin. Bundesgesundheitsblatt52:851-861 
4. Bierbaum T (2016) Potentialorientierte Karriereförderung wissenschaftlichen Nachwuchses in der Life Sciences im interkulturellen Spannungsfeld am Beispiel des BMEP. Dissertation Dr. rer. biol. hum. Medizinische Hochschule Hannover, Hannover

5. Bitter-Suermann D (2009) Promovieren in der Medizin. Ein Plädoyer für den studienbegleitenden Dr. med. Forsch Lehre 2009:580-581

6. Bitzinger D, Heberlein A, Theilmeier G (2014) Die Generation $Y$ will klare Aussagen über Karrierewege. Dtsch Ärztebl 111:B 384-B 386

7. Buddeberg-Fischer B, Illés C, Klaghofer R (2002) Karrierewünsche und Karriereängste von Medizinstudierenden - Ergebnisse von Fokusgruppeninterviews mit Staatsexamenskandidatinnen und -kandidaten. Gesundheitswesen 64:353-362

8. Buddeberg-Fischer B, Stamm M (2010) The medical profession and young physicians lifestyle in flux: Challenges for specialty training and health care delivery systems. Swiss Med Wkly:. doi:10.4414/ smw.2010.13134

9. Deutsche Forschungsgemeinschaft (2012) Förderatlas 2012, Kennzahlen zur öffentlichen finanziellen Förderung in Deutschland. Deutsche Forschungsgemeinschaft, Bonn

10. Deutsche Forschungsgemeinschaft (2014) Ständige Senatskommission für Grundsatzfragen in der Klinischen Forschung der Deutschen Forschungsgemeinschaft. Deutsche Forschungsgemeinschaft, Bonn

11. Deutsche Forschungsgemeinschaft (2014) GEPRIS, Geförderte Projekte der DFG. Deutsche Forschungsgemeinschaft, Bonn

12. Dzwonnek D (2014) Gefahr oder Garant? Drittmittelforschung und Forschungsfreiheit - Anmerkungen zu einem unvermuteten Zusammenhang. Forsch Lehre 2:92-94

13. Else Kröner Fresenius Stiftung (2014) Rahmenbedingungen medizinischer Forschung in Deutschland. Else Kröner Fresenius Stiftung, Bad Homburg

14. Epstein N, Pfeiffer M, Eberle J, von Kotzebue L, Marins T, Lachmann D, Mozhove A, Bauer J, Berberat PO, Landmann M, Herzig S, Neuhaus BJ, Offe K, Prenzel M, Fischer MR (2016) Nachwuchsmangel in der medizinischen Forschung. Wie kann der ärztliche Forschernachwuchs besser gefördert werden? Beitr Hochschulforsch 38:162-187

15. Expertenkommission (2014) Forschung und Innovation (EFI) Berlin 54-84.

16. Fischer MR (2012) Medizinstudium als Grundstein für Versorung und Forschung. Dtsch Ärztebl 109:325-326

17. Flintrop J (2013) Finanzierung der Hochschulmedizin: „Es ist fünf vor zwölf“. Dtsch Ärztebl 11:C 1732

18. Hermann-Lingen C, Brunner E, Hildenbrand S et al (2014) Evaluation of medical research performance-position paper of the Association of the Scientific Medical Societies in Germany (AWMF). GMS Germ Med Sci 12:B0C1

19. Krömer HK, Kronczak M (2014) Forschung braucht Infrastruktur. Dtsch Ärztebl 111:B 150-B 151

20. Miemietz B (Hrsg) (2012) Das Ina PichlmayrMentoring für Nachwuchswissenschaftlerinnen an der Medizinischen Hochschule Hannover. Einblicke in das Programm. Medizinische Hochschule Hannover, Hannover

21. Milewicz M, Lorenz G, Dermody S, Brass F, The National Association of MD-ÖPhD Programs Executive Committee (2015) Rescuing the physicianscientist workforce: The time for action is now. JClin Invest 125:3742-3747
22. Pabst R, ParkDH, Paulmann V(2012)DiePromotion in der Medizin ist besser als ihr Ruf. Ergebnisse einer Befragung von Doktoranden. Dtsch Med Wochenschr 137:2311-2315

23. Pabst $R$, Linke $P$ (2014) Junge Mediziner fördern. Eine Anschubfinanzierung der Forschung. Forsch Lehre 21:582-583

24. Paulmann V, Fischer V, Dudzinska A, Pabst R (2015) Chirurgie als Weiterbildungsfach für Ärztinnen. Ergebnisse von Lehrevaluationen und Absolventenstudien an der $\mathrm{MHH}$. Chirurg 86:595-602

25. Pfeiffer M, Fischer MR, Bauer D (2016) Publication activities of German junior researchers in academic medicine: Which factors impact impact factors? BMC Med Educ 16:190. doi:10.1186/s12909-0160712-3

26. Schmidt CE, Möller J, Schmidt K, Gerbershagen MU, Wappler F, Limmroth V, Padosch SA, Bauer M (2011) Generation Y: Rekrutierung, Entwicklung und Bindung. Anästhesist 60:517-524

27. Sorg H, Betzler C, Grieswald C, Schwab CGG, Tilkorn DJ, Hauser J (2016) Die medizinische Habilitation: akademische Befähigung oder Karriereinstrument. Chirurg. doi:10.1007/s00104015-0131-8
Innerklinische Notfallteams müssen früher intervenieren

In Deutschlands Kliniken kommt es jedes Jahr zu rund 93.000 Herz-Kreislaufstillständen. Daten des Dresdner Uniklinikums belegen: Ein frühzeitiger Einsatz innerklinischer Notfallteams senkt die Zahl von HerzKreislaufstillständen und erhöht die Überlebensrate.

Allerdings wird derzeit lediglich ein Fünftel aller in deutschen Krankenhäusern tätigen innerklinischen Notfallteams (Medical Emergency-Teams - MET) bereits bei Warnsymptomen aktiv, wie eine Umfrage der Anästhesisten des Uniklinikums ergab.

Damit die Ärzte und das Pflegepersonal des Dresdner Klinikums die frühen Anzeichen einer lebensbedrohlichen Situation von Patienten zuverlässig erkennen können, haben sie einen Kriterienkatalog erstellt und schulen die Mitarbeiter der Stationen regelmäßig. Dazu gehört auch ein obligatorisches jährliches klinikinternes Reanimationstraining.

Zudem wurden die Notfallequipments vereinheitlicht, automatisierte externe Defibrillatoren angeschafft sowie die Logistik der Alarmierung und des Transports verbessert. So konnte erreicht werden, dass die Zahl der notwendigen Reanimationen trotz steigendem Schweregrad und paralle steigender Patientenzahl stabil geblieben ist.

Auch die Rate der reanimierten Patienten, die 30 Tage nach der Entlassung noch leben, ist am Klinikum dreimal so hoch wie im Bundesdurchschnitt. Es bestehe die realistische Chance, in Deutschland jedes Jahr das Leben von rund 30.000 Krankenhauspatienten zu retten, resümieren die Ärzte.

Quelle: Universitätsklinikum Carl Gustav Carus Dresden www.uniklinikum-dresden.de 